\title{
Validity of FDG PET/CT in patients presenting with primary colonic symptoms
}

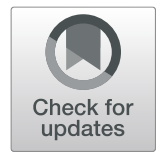

\author{
Gihan Hassan Gamal
}

\begin{abstract}
Background: The usefulness of FDG PET/CT has been shown for staging and restaging of CRC patients. The purpose of this study was to determine the accuracy of 18F-FDG PET/CT in the detection of advanced colorectal adenomas in screening of high-risk asymptomatic individuals.

Results: A prospective study was conducted on 500 patients from January 2016 to June 2018 (300 male, 200 female) above 50 years of age (mean age 67.5 years) with +ve family history of CRC who underwent CTC. PET/CT was performed to all patients with +ve CTC findings.

PET/CT sensitivity, specificity, positive predictive value, negative predictive value, and accuracy for all carcinomas and adenomas with high-grade dysplasia, per lesion, were $94 \%, 35 \%, 85 \%, 62 \%$, and $66 \%$ respectively. PET/CT sensitivity, specificity, positive predictive value, negative predictive value, and accuracy for all carcinomas and adenomas with high-grade dysplasia, per patient, were $91 \%, 80 \%, 89 \%, 83 \%$, and $86 \%$ respectively. Histopathology results were used as standard of reference.
\end{abstract}

Conclusions: 18F-FDG-PET/CT is a promising tool in detecting advanced adenomas or carcinomas in high risk patients and it additionally enhance compliance with a screening program.

\section{Background}

Colorectal cancer is the third most diagnosed cancer in men, next to prostate and lung carcinoma. In women, it is the second most diagnosed cancer, next to breast cancer [1]. Studies have shown many risk factors which may increase the chance of developing $\mathrm{CRC}$, as age above 50 years, individuals with positive family history of colorectal cancer [2]. Individuals with other risk factors as hereditary colorectal neoplasia require special surveillance programs, a description of which is beyond the scope of this study.

Over the years, screening in asymptomatic moderate to high-risk patients is getting more beneficial for reducing CRC and led to sharp decline in CRC mortality rates. Premalignant lesions are the main target lesions for CRC screening, as advanced adenomas with a size $=$ or $>10 \mathrm{~mm}$, adenomas with high-grade dysplasia and carcinomas [3].

Previous studies depicted that the early diagnosis and removal of premalignant adenomas may prevent development of CRCs and could substantially help in decrease CRC mortality rate. There is a firm consensus

Correspondence: dr.gh_006@yahoo.com

Department of Radiodiagnosis October 6 University, Giza, Egypt that larger ( $>$ or $=10 \mathrm{~mm}$ ) colonic adenomas should be removed; however, smaller one with a size $<10 \mathrm{~mm}$ shows low prevalence of developing high-grade dysplasia or transforming into carcinoma. So, the importance of its removal is more controversial [4].

18F-FDG PET/CT as a noninvasive, painless molecular imaging technology which provides both functional and anatomical data correlates with the grading and the degree of malignancy of carcinoma. Although the usefulness of this technique has been established for staging and restaging of CRC patients, its valuability as a screening tool in high risk patients is still controversial $[5,6]$.

The purpose of this study was to determine the accuracy of 18F-FDG PET/CT in the detection of advanced colorectal adenomas in screening of high-risk asymptomatic individuals.

\section{Patients and methods}

A prospective study was conducted on 500 patients from January 2016 to June 2018 (300 male, 200 female) above 50 years of age (mean age 67.5 years) with +ve family history of CRC who underwent CTC. PET/CT was performed to all patients with +ve CTC findings. 
Patients with a fasting glucose level $200 \mathrm{mg} / \mathrm{dL}$ were excluded (exclusion criteria). Histopathology results were used as standard of reference.

\section{The PET/CT technique}

PET/CT scan was performed using Siemens Biograph TruePoint 64 (Siemens Healthcare, Erlangen, Germany).

All patients were asked to fasten for at least $5 \mathrm{~h}$ to lower the insulin and blood glucose level. Blood glucose levels were in its normal levels prior to 18-FDG injection. PET images were done $60 \mathrm{~min}$ after I.V injection of $5 \mathrm{MBq} / \mathrm{kg}$ body weight of FDG (up to $550 \mathrm{MBq}$ ).

The standard uptake value (SUV) was defined as the tissue concentration $(\mathrm{MBq} / \mathrm{mL})$ of the tracer divided by the activity injected per body weight $(\mathrm{MBq} / \mathrm{g})$. The maximum SUV in the volume of interest was considered as the SUVmax for the purpose of analysis. Scans were acquired from skull vertex down to the thigh. Prior to PET imaging, a diagnostic CT scan of the brain, neck, chest, abdomen, and pelvis was performed with oral and without I.V contrast for attenuation correction and anatomic localization. The following parameters were used: $80-$ $100 \mathrm{~mA}, 140 \mathrm{kV}, 5 \mathrm{~mm}$ collimation, $0.5 \mathrm{~s}$ rotation time, and pitch $=0.984$. Patients were in supine position with both arms up; normal respiration was maintained during the scanning. Reconstruction with section thickness of $1.2 \mathrm{~mm}$ was done. Subsequently, PET data were acquired in at 5-7 bed positions. 18FDG-PET images were reconstructed; 18FDG-PET, CT, and fused 18FDG-PET/CT images were reviewed on the dedicated workstation. Patients were asked to have FDG PET/CT and CTC imaging performed within 3 days of both examinations.

The PET/CT images were interpreted by an experienced radiology consultant, and reports were compared to those of CT colonography. The reader was not blinded to other modality results.

\section{CT colonography technique}

CT colonography was performed after full bowel preparation. MDCT (Toshiba 320-detector CT Medical System) was used with a maximum detector collimation of $2.5 \mathrm{~mm}$, pitch factor $2: 1,200 \mathrm{mAs}, 120-150 \mathrm{kV}$, matrix $512 \times 512$, full field of view $40 \mathrm{~cm}$. Scanning time was done in one breath hold $(20 \mathrm{~s})$. All patients were examined cranio-caudally starting from the level of the diaphragmatic copula down to the anus. Readers used 2D and $3 \mathrm{D}$ visualization. CTC was carried out by an experienced radiology consultant.

The FDG PET/CT findings were classified as follows:

True positive findings were occurrence of focal hypermetabolic lesions in a compatible site of advanced adenomas on CTC. False positive findings were occurrence
Table 1 PET/CT sensitivity, specificity, positive predictive value, negative predictive value, and accuracy for all carcinomas and adenomas with high-grade dysplasia per lesion

\begin{tabular}{ll}
\hline Sensitivity & $94 \%$ \\
Specificity & $35 \%$ \\
NPV & $85 \%$ \\
PPV & $62 \%$ \\
Accuracy & $66 \%$ \\
\hline
\end{tabular}

of focal hypermetabolic lesions with no evidence of advanced adenomas in a compatible site on CTC.

False negative findings were lesions which did not display focal hypermetabolic lesions on PET/CT but with positive evidence on CTC.

\section{Statistical analysis}

The FDG PET/CT sensitivity, specificity, positive predictive value, negative predictive value, and lastly the overall accuracy of colorectal neoplasms detection were calculated. Mc Nemar and T test were used to compare the results, with statistical significance $p<0.05$. The standard maximum uptake value (SUVmax) of each focal hypermetabolic lesion was quantitative and qualitative evaluated.

\section{Results}

A prospective study was conducted on 500 patients from January 2016 to June 2018 (300 male, 200 female), above 50 years of age (mean age 67.5 years) with +ve family history of CRC who underwent CTC. PET/CT was performed to all patients with +ve CTC findings.

Histopathology results were used as standard of reference. Among the 500 CTC examinations, 142 colonic neoplastic polyps and 19 colonic carcinomas were detected by CTC. Histological examination of the adenomas revealed 3\% (4/142) show no dysplasia, 69\% (98/ $142)$ with low-grade dysplasia, and $28 \%$ (40/142) show high-grade dysplasia. ADR (adenoma detection rate) revealed $28.4 \%$. The prevalence rate of colonic carcinoma detection was $3.8 \%$.

Table 2 PET/CT sensitivity, specificity, positive predictive value, negative predictive value, and accuracy for all carcinomas and adenomas with high-grade dysplasia per patient

\begin{tabular}{ll}
\hline Sensitivity & $91 \%$ \\
Specificity & $80 \%$ \\
NPV & $89 \%$ \\
PPV & $83 \%$ \\
Accuracy & $86 \%$ \\
\hline
\end{tabular}




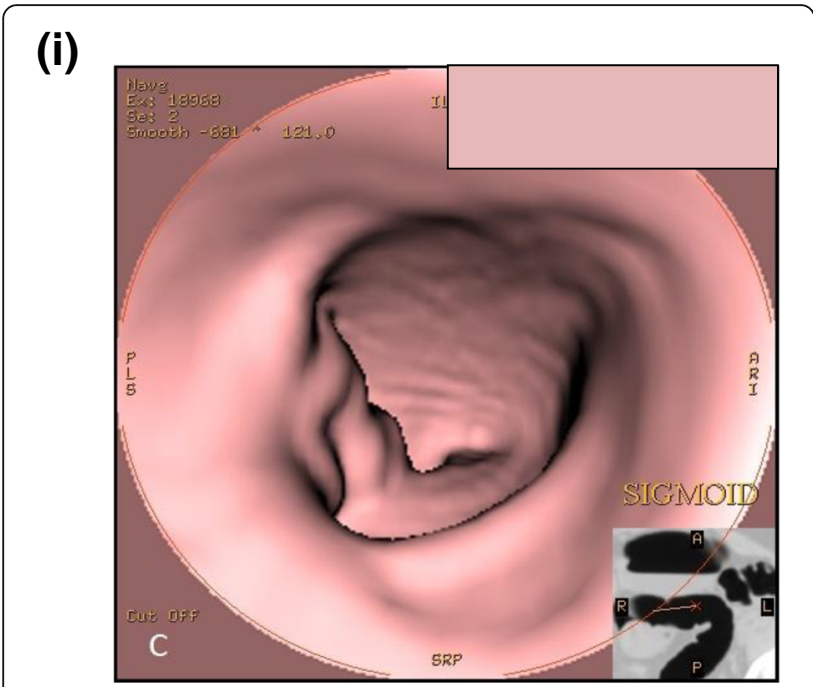

(ii)
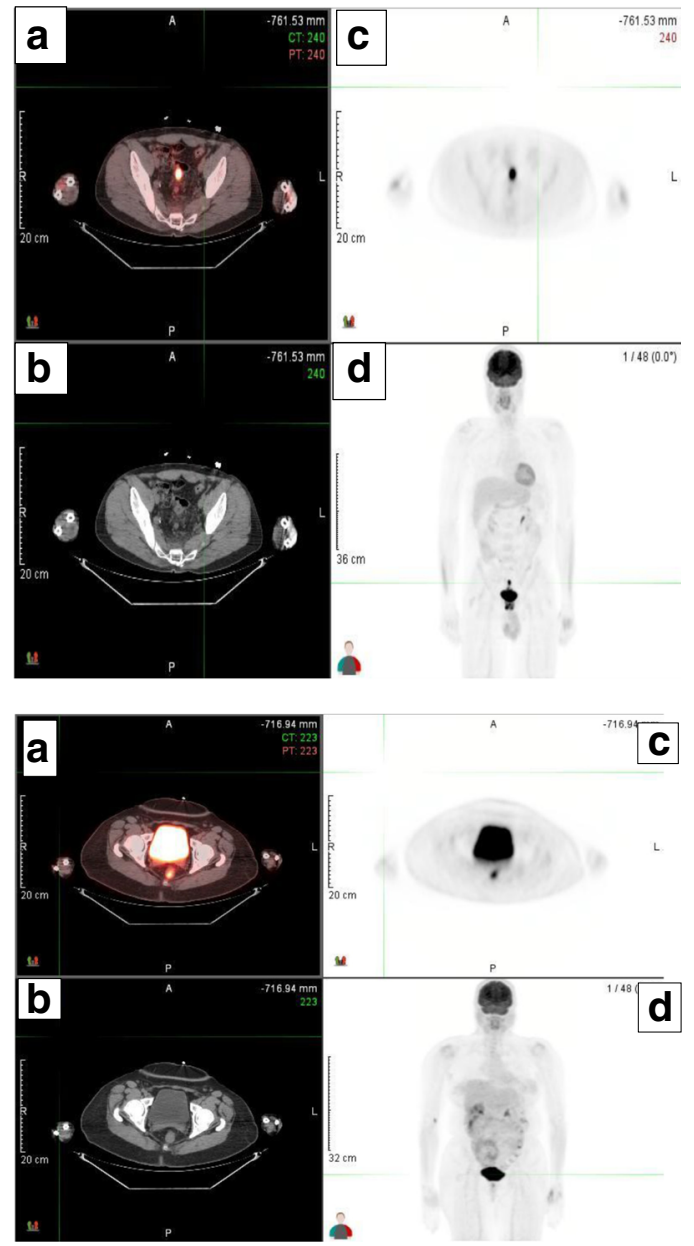

Fig. 1 A 69-year-old male. i CT colonography revealed a rectosigmoid polyp. ii FDG avid pathologically proved high-grade dysplastic rectosigmoid polyp with SUVmax 14.2. a Axial PET/CT. b Axial CT. c, d PET
Avid PET/CT for relevant masses were present in $90 \%$ of all correlated masses (145/161), 83\% for relevant adenomas $(118 / 142)$ with size range of $9-25 \mathrm{~mm}$ and $100 \%(19 / 19)$ for carcinomas with size range of $8-80 \mathrm{~mm}$. PET/CT failed to visualize $10 \%$ of relevant masses $(16 / 161)$. All masses not visualized on PET/CT were adenomas $<6 \mathrm{~mm}$ in size or adenomas $\geq 10 \mathrm{~mm}$ but with low-grade dysplasia.

False-positive FDG uptakes were 15\% of cases (24/161); they include inflammatory lesions, ulcerative colitis, and benign adenomas.

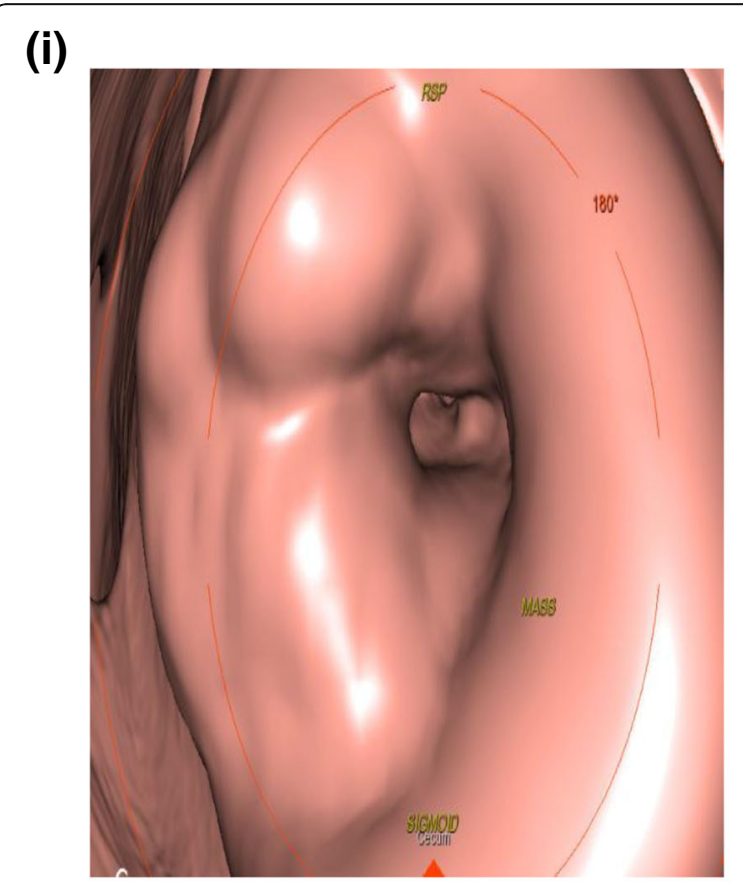

(ii)

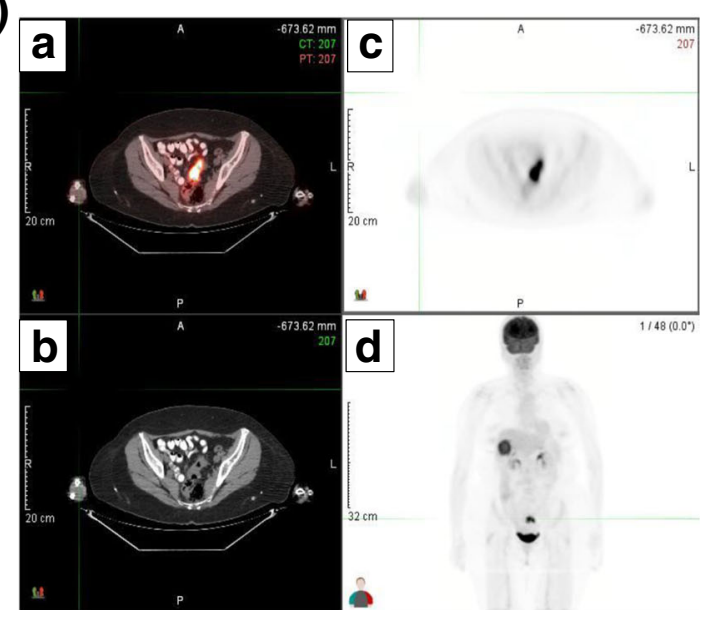

Fig. 2 A 76-year-old female. i CT colonography revealed 5-cm-long segment of concentrically thickened mid to distal sigmoid colon. ii FDG avid 5-cm-long segment of concentrically thickened mid to distal sigmoid colon. Pathologically proved sigmoid carcinoma with SUVmax 13.7. a Axial PET/CT. b Axial CT. c, d PET 
The mean and standard deviation of SUVmax of different lesions were as follows: Lesions with lowgrade dysplasia $=9.7+-6.9$, with high-grade dysplasia $=11.6+-4.1$. The mean and standard deviation of SUVmax of carcinomas were $12.9+-6.8$ while those of false +ve uptakes were $7.4+-3.8$

$\mathrm{PET} / \mathrm{CT}$ sensitivity, specificity, positive predictive value, negative predictive value, and accuracy in detection of advanced adenoma and carcinomas were showed in Tables 1 and 2).

\section{Discussion}

In CRC patients, PET/CT has been proved to be of a great value in staging and restaging of tumors. However, its value and usefulness as a screening tool in moderate to high-risk patients for colorectal tumors remains an open question. Studies have reported a wide range of CRC tumors detection 20 to $90 \%$. There are many factors that contribute in this discrepancy, such as using of different screening protocols, gold standards, variable inclusion criteria, different time gap between the imaging tools, and the variability between the images readers [7-9].

Our data revealed the detection rates of adenomas and colorectal carcinomas were $28.4 \%$ and $3.8 \%$ respectively.

Furthermore, our results demonstrate that focal hypermetabolic lesions on PET/CT images correlated with $90 \%$ of all CTC masses (145/161), 83\% with relevant adenomas $(118 / 142)$ with size range of $9-25 \mathrm{~mm}$ and $100 \%(19 / 19)$ for carcinomas with size range of $8-80 \mathrm{~mm}$.

These rates are in accordance to those previously reported for screening of asymptomatic high to average risk patients which concluded that the detection of colorectal neoplasms by FDG PET/CT are positively correlated with the size and the degree of dysplasia of the lesion [10, 11]. In our study, the mean and standard deviation of SUVmax of low-grade dysplasia was $9.7+-6.9$ and that of high-grade dysplasia was $11.6+-4.1$ (Figs. 1 and 2).

In addition, PET/CT has failed to visualize $10 \%$ of relevant masses (16/161) and the probable explanation was that all masses were adenomas $<8 \mathrm{~mm}$ in size or adenomas $\geq 10 \mathrm{~mm}$ but with low-grade dysplasia (Fig. 3). The presence of low-grade dysplasia of the lesion has been found to be significantly a cause for a low likelihood of positive PET/CT imaging findings.

False-positive FDG uptakes were found in this study in 24/161 of PET/CT images, which included inflammatory lesions, ulcerative colitis, and benign adenomas. These figures are similar with those previously reported in the literature [10].

All carcinomas were visualized by PET/CT (19/19) $100 \%$ with size range $8-80 \mathrm{~mm}$ and the mean and standard deviation of SUVmax $=12.9+-6.8$. These were in accordance with the literature which reported SUVmax $=12.6+-4.9(n=51)[12,13]$.

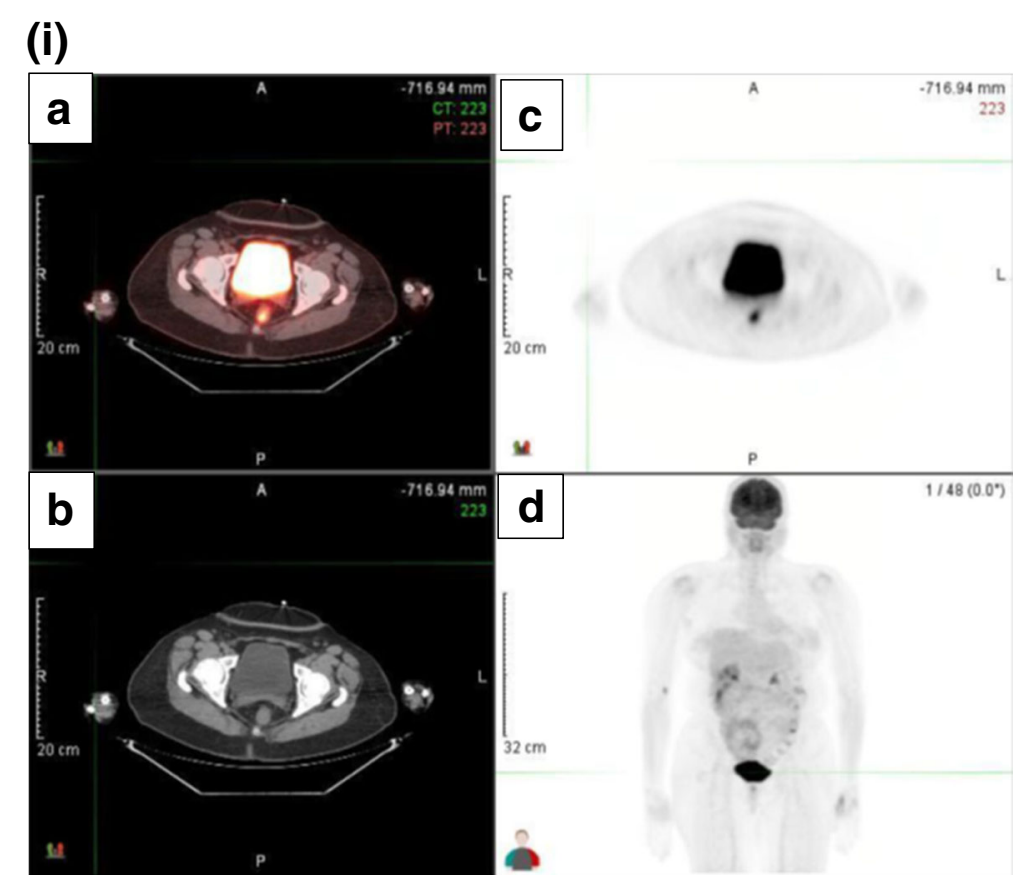

Fig. 3 A 55-year-old female. (i) Physiological mild nonfocal rectal activity. SUVmax 5.9, pathologically proved a false +ve case. a Axial PET/CT. b Axial CT. c, d PET 
Our study has faced several limitations: first, high cost and, second, the small number of participants with a wide range of population, so the rate of hypermetabolic neoplasms detection on PET/CT imaging was low.

\section{Conclusions}

Early prediction of cancer based on FDG PET/CT imaging has prognostic and predictive significance in patients with high risk of developing cancer.

Thus, PET/CT as a non-invasive method may be a promising tool in detecting advanced adenomas or carcinomas and additionally enhance compliance with a screening program.

\section{Abbreviations}

18F-FDG PET/CT: 18F-Fluorodeoxyglucose positron emission tomography/ computed tomography; CRC: Colorectal cancer; CTC: Computed tomography colonography; SUV: Standard uptake value

\section{Acknowledgements}

Not applicable for that section.

\section{Author's contributions}

The author read and approved the final manuscript.

\section{Funding}

Not applicable for that section

\section{Availability of data and materials}

Not applicable for that section.

\section{Ethics approval and consent to participate}

The protocol of the current study was approved by the ethical scientific committee of the institutional review board. Written consent was signed prior to perform the examination for each patient.

\section{Consent for publication}

Not applicable for that section.

\section{Competing interests}

The author declare that he/she has no competing interests.

Received: 6 June 2019 Accepted: 2 July 2019

Published online: 05 August 2019

\section{References}

1. Jemal A, Bray F, Center MM, Ferlay J, Ward E et al (2011) Global cancer statistics. CA Cancer J Clin 61:69-90

2. Ferlay J, Soerjomataram I, Ervik M, Dikshit R, Eser S, Mathers C (2013) GLOBOCAN 2012 v1.0, Cancer Incidence and Mortality. Worldwide: IARC CancerBase No. 11 [Internet]. International Agency for Research on Cancer, Lyon, France

3. Gollub MJ et al (2014) Diagnostic accuracy of 18F-FDG PET/CT for detection of advanced colorectal adenoma. Clin Radiol 69(6):611-618.

4. National Comprehensive Cancer Network, Clinical Practice Guidelines in Oncology (NCCN Guidelines) Colon Cancer, version 2.2016.

5. van de Velde CJH, Boelens PG, Borras JM et al (2014) EURECCA colorectal: multidisciplinary management: European consensus conference colon \& rectum. Eur J Cancer 50:1.e1-1.e34

6. Huang S-W, Hsu C-M, Jeng W-J, Yen T-C, Su M-Y et al (2013) A Comparison of positron emission tomography and colonoscopy for the detection of advanced colorectal neoplasms in subjects undergoing a health check-up. PLoS ONE 8(7):e69111. https://doi.org/10.1371/journal.pone.006911

7. Yapar Z, Kibar M, Yapar AF et al (2010) The value of 18Ffluorodeoxyglucose positron emission tomography/computed tomography in carcinoma of an unknown primary: diagnosis and follow-up. Nucl Med Commun 31:59-66
8. Ozis SE, Soydal C, Akyol C et al (2014) The role of 18F-fluorodeoxyglucose positron emission tomography/computed tomography in the primary staging of rectal cancer. World J Surg Oncol 12:26-33

9. Petersen RK, Hess S, Alavi A, Høilund-Carlsen PF (2014) Clinical impact of FDG-PET/CT on colorectal cancer staging and treatment strategy. Am J Nucl Med Mol Imaging 4(5):471-482

10. Wolfgang $L$, Teresa $V$, Baerbel $W$ et al (2010) Detection of relevant colonic neoplasms with PET/CT: promising accuracy with minimal CT dose and a standardised PET cut-off. Eur Radiol 20(9):2274-2285. Published online 2010 May 26. https://doi.org/10.1007/s00330-010-1772-0

11. Peng J, He Y, Xu J, Sheng J, Cai S et al (2011) Detection of incidental colorectal tumours with 18F-labelled 2-fluoro-2-deoxyglucose positron emission tomography/computed tomography scans: results of a prospective study. Colorectal Dis 13:e374-e378

12. Chen YK, Kao CH, Liao AC, Shen YY, Su CT (2003) Colorectal cancer screening in asymptomatic adults: the role of FDG PET scan. Anticancer Res 23:4357-4361

13. Gutman F, Alberini JL, Wartski M et al (2005) Incidental colonic focal lesions detected by FDG PET/CT. AJR Am J Roentgenol 185:495-500

\section{Publisher's Note}

Springer Nature remains neutral with regard to jurisdictional claims in published maps and institutional affiliations.

\section{Submit your manuscript to a SpringerOpen ${ }^{\circ}$ journal and benefit from:}

- Convenient online submission

- Rigorous peer review

- Open access: articles freely available online

- High visibility within the field

- Retaining the copyright to your article

Submit your next manuscript at $>$ springeropen.com 